\title{
Germanica
}

\section{Stig Dagerman ou les incertitudes d'un engagement}

Stig Dagerman, oder das unschlüssige Engagement

\section{Georges Ueberschlag}

\section{CpenEdition}

\section{Journals}

Édition électronique

URL : http://journals.openedition.org/germanica/2099

DOI : 10.4000/germanica.2099

ISSN : 2107-0784

\section{Éditeur}

Université de Lille

\section{Édition imprimée}

Date de publication : 1 janvier 1992

Pagination : 135-153

ISSN : 0984-2632

\section{Référence électronique}

Georges Ueberschlag, "Stig Dagerman ou les incertitudes d'un engagement », Germanica [En ligne], 10 | 1992, mis en ligne le 12 février 2014, consulté le 06 octobre 2020. URL : http://

journals.openedition.org/germanica/2099 ; DOI : https://doi.org/10.4000/germanica.2099

Ce document a été généré automatiquement le 6 octobre 2020.

(c) Tous droits réservés 


\title{
Stig Dagerman ou les incertitudes d'un engagement
}

\author{
Stig Dagerman, oder das unschlüssige Engagement
}

Georges Ueberschlag

1 Il arrive a des personnes, qui sont souvent des personnages, dans un moment d'égarement ou au contraire dans un moment d'extrême lucidité, de retour sur soi, un moment où l'on fait le bilan, de rédiger déjà de leur vivant l'épitaphe qui devra un jour être inscrite sur leur pierre tombale, pensant sans doute s'assurer ainsi sinon une gloire posthume, du moins une longue vie dans la mémoire des hommes.

2 Stig Dagerman, chose assez surprenante de sa part, s'est essayé lui aussi à cet exercice. En effet, au mois de septembre 1951, trois années avant sa mort, très déprimé, il écrit à son éditeur Ragnar Svanström, qui fut aussi son ami et confident, pour lui faire part de sa sécheresse et de son incapacité de produire quoi que ce soit, étant en proie à une sorte de paralysie intellectuelle.

"Que ce soit ou non le fait d'une maladie d'enfance, écrit-il, je ne suis pas capable de grand-chose actuellement et je ne peux rien contre la nausée qui me prend ni contre la fatigue d'écrire $»^{1}$. Et il joint à sa lettre une petite feuille à part, sur laquelle il vient de jeter le résultat de ses méditations au cours d'une promenade en montagne. C'est une sorte de conclusion à laquelle il aboutit, qui ne fait d'ailleurs que reprendre d'autres méditations antérieures, une sorte de point final qu'il met au chapitre de sa vie :

Je quitte et des rêves immuables et des liaisons instables... Je quitte et une mauvaise réputation et la promesse d'une réputation pire encore. Je quitte quelques centaines de milliers de mots, certains écrits avec plaisir, la plupart écrits avec ennui et pour de l'argent. Je quitte une position irrésolue face aux problèmes de notre temps, un doute qui a déjà trop servi, mais de bonne qualité, et l'espoir d'une délivrance.

J'emporte dans mon voyage une connaissance inutile du globe... et un amour malheureux pour la jeunesse européenne. J'emporte enfin la vision d'une pierre tombale qui s'élève dans le désert ou au fond de la mer et qui porte cette inscription : 
Ci-gît un écrivain suédois

tombé pour rien,

Son crime était l'innocence,

Oubliez-le souvent ${ }^{2}$.

4 Le ton de cette missive et de cette ébauche d'épitaphe, qui était d'ailleurs un pastiche à peine déguisé d'un poème assez célèbre d'Erik Blomberg écrit pour rappeler la mémoire des ouvriers suédois tombés sous les balles lors des grèves d'Ådalen en 1931, est assez amer et angoissé, sinon résigné déjà, malgré son humour un peu macabre, un ton d'abandon qui jette une lumière singulière sur notre propos, sur l'impossibilité qu'il y a eu pour Stig Dagerman de réaliser son ambition, ses «rêves immuables » comme il le dit, et sur l'ambiguïté de son engagement, de sa "position irrésolue» face aux problèmes de notre temps, qu'il avait voulu prendre à bras-le-corps.

Et pourtant, n'est-il pas célèbre, entouré de la considération générale? Ses éditeurs, son public attendent beaucoup de lui. Il n'a pas encore trente ans. Il vient de rencontrer une jeune actrice alors très célèbre en Suède elle aussi, et très belle, Anita Björk, qui a été la magnifique interprète de Mademoiselle Julie dans le film d'Alf Sjöberg. Il en est violemment épris, du moins à en croire ses amis, et bientôt il va l'épouser, après son divorce d'un premier mariage.

6 Alors pourquoi ce désenchantement de jeunesse, ce spleen et ce mal de vivre? Nous savons bien aujourd'hui comment finira l'histoire de Stig Dagerman. Le 3 novembre 1954 il va porter son billet quotidien, son dagsedel, à la rédaction du journal Arbetaren, qui est l'organe du mouvement anarcho-syndicaliste auquel il collabore depuis 1943 et dont il a dirigé pendant un certain temps la page littéraire. Il a fait son travail de journaliste, il a livré son billet du jour - il y en a déjà eu plus de mille avant celui-ci - un billet de révolte au quotidien. Puis il retourne à la villa qu'il occupe avec Anita Björk, une villa cossue dans un beau quartier du grand Stockholm, va s'enfermer dans son garage pour se donner la mort en laissant tourner le moteur de sa voiture.

7 Suicide réussi, après plusieurs tentatives antérieures qui l'avaient mené, dans une attitude de défi, de plus en plus près du bord et du seuil de non-retour.

Par quel mal a-t-il été vaincu, et pourquoi cette obsession suicidaire, ce désir de mourir, qui s'amplifient dans les derniers mois de son existence et auxquels une tentative de traitement psychiatrique n'avait pas apporté de réponse, le patient s'étant enfermé dans son mutisme et dans une attitude de refus?

Stig Dagerman se trouve alors dans une position de plus en plus inconfortable, intellectuellement. Il a le sentiment d'être tiraillé, écartelé entre des exigences, des obligations, des besoins contradictoires, et cela le paralyse progressivement. Lui, l'écrivain célèbre, au génie ardent et impatient, ne produit plus rien - «je ne peux rien contre la nausée qui me prend ni contre la fatigue d'écrire»- il n'est plus que journaliste, c'est-à-dire un écrivain pris dans les contingences du temps présent et qui essaie de pratiquer un art auquel il se sent obscurément allergique, dont il a dit, en critique lucide et affectueux, que c'était l'art d'arriver en retard aussi tôt que possible, ajoutant même : un art que je n'apprendrai jamais.

10 Le drame se noue donc avec cette lenteur inexorable avec laquelle Stig Dagerman s'avance dans la conscience de sa stérilité. Pour le comprendre il faut partir, pensonsnous, de ce qui a été à la base même de la vocation d'écrivain de Dagerman, de ce qui a été sa raison d'être, même en tant qu'homme, à savoir son engagement, son engagement politique primordial. 
Stig Dagerman s'était engagé bien avant d'écrire. Il voulait corriger le monde, et son activité d'écrivain n'a été qu'un moyen, celui qui lui convenait le mieux, pour ne pas dire le seul qui lui convenait, pour agir sur la société.

Son engagement ne l'a cependant conduit à aucun mandat électif, si ce n'est celui, bien éphémère, de secrétaire de son club syndical. Il n'avait pas le tempérament d'un homme politique, de quelqu'un qui brigue des mandats. Non pas qu'il ait eu le moindre dédain pour la vie politique en soi, qu'il ne confondait pas avec les politiciens de la neutralité et des compromissions qu'il a tant dénoncés en Suède. Mais il lui manquait pour cela deux qualités essentielles. Restant obstinément victime de sa timidité naturelle, il n'avait pas le sens du contact, et le don de la parole publique lui faisait complètement défaut. Les quelques rares fois où il s'est hasardé à faire des discours publics, lors de meetings, il donnait une telle impression d'insécurité, d'être mal à son aise, ayant tout rédigé, ne laissant aucune place à l'improvisation, qu'il devenait bien vite évident que l'expression écrite sera son seul recours, une expression concise, juste et pertinente, sans inutiles artifices oratoires, allant droit aux choses, une écriture qui sera celle d'un visiteur pressé et inquiet qui ne saura parler que de l'essentiel.

Engagé dans le syndicalisme grâce à son père, et bientôt dans l'anarcho-syndicalisme, Stig Dagerman, qui avait de l'ambition et qui avait le talent de l'écriture, s'oriente donc tout naturellement vers une activité de la plume. Il devient journaliste.

Mais pour un journaliste anarcho-syndical une carrière politique était à peu près totalement exclue. Il ne pouvait que rester à l'écart, surtout dans une Suède timorée, assise fiévreusement sur sa neutralité. Or, les premiers succès de Dagerman comme homme de lettres, le succès de son premier roman surtout, Ormen (Le Serpent), publié en 1945 et accueilli avec enthousiasme par une critique unanime, le troublent profondément, en ce sens qu'ils lui révèlent la possibilité d'une autre voie pour agir sur le monde, pour sortir de l'ombre et porter ses idées sur la place publique. Ils lui révèlent que sa détermination de devenir écrivain, c'était le bon choix. Mais ce choix de la littérature n'était pas sans équivoque ni sans danger, un danger qu'il nous faudra analyser, car Dagerman continuait à placer au-dessus de tout son désir de travailler à la réalisation de son idéal libertaire et anarchiste.

5 Le compromis qu'il pense un moment avoir trouvé, en tant qu'écrivain anarchiste, n'est malgré tout qu'un compromis, même lorsqu'il se qualifie lui-même de «politicien de l'impossible ».

Dans un petit essai intitulé L'Anarchisme et moi, il écrit en effet :

L'écrivain anarchiste peut pour l'instant s'attribuer le rôle modeste du ver de terre dans l'humus culturel... Être le politicien de l'impossible dans un monde où ceux du possible ne sont que trop nombreux, est malgré tout un rôle qui me satisfait à la fois comme être social, comme individu et comme auteur du Serpent ${ }^{3}$.

Ce texte date de 1946, il a donc été écrit un an à peine après la publication du Serpent, en pleine gloire naissante. Mais la suite prouvera l'impossibilité pour Stig Dagerman de s'y tenir, même de se satisfaire, comme il le dit, de ce rôle modeste, et donc l'impossibilité d'assumer la part d'ambiguïté qui s'insinuera progressivement dans son engagement, à l'analyse de laquelle nous tenterons d'apporter quelques éléments de réponse, en 
restant conscient, bien sûr, du mystère qui entoure toute création et en restant modestement en deçà du seuil de ce mystère. que n'ayant aucune qualité particulière de travailleur manuel, ni même la vocation d'écrivain prolétarien. Prenant conscience de ce fait, il a essayé de lutter contre ce sentiment d'exclusion de son milieu naturel. Après son baccalauréat, par exemple, il a tout de suite voulu rechercher un emploi. L'université ne le tente pas, ne serait-ce qu'à cause du danger de le rendre encore plus étranger à sa classe. Il veut continuer à se sentir laborieux avec les laborieux, humble avec les humbles, malgré son orgueil. Mais ce sera dans le cadre bien particulier des groupements dans lesquels son père l'avait introduit et des journaux anarcho-syndicalistes. Son désir d'intégration au monde ouvrier trouve là un succédané, une sorte de fuite en avant.

Entré le 25 février 1941 dans les rangs de la jeunesse anarcho-syndicaliste, à dix-huit ans, il est parfaitement conscient du sens de son engagement : mettre son idéalisme au service d'une cause, celle de la liberté de l'homme. En revanche, la poursuite de cet engagement, la persévérance dans la voie du militantisme, cela sera beaucoup moins évident pour Stig Dagerman et n'ira pas sans problèmes. Ayant opté pour un mouvement révolutionnaire en opposition complète avec les orientations traditionnelles de la société suédoise, héritière de la société oscarienne du XIX siècle, il s'y fait très vite remarquer par ses talents, ses observations pleines d'humour et de causticité, et il devient le secrétaire de son club. Cependant, il n'a rien d'un théoricien ni d'un doctrinaire, même si la plume devient sa seule arme et l'expression écrite le seul moyen pour lui de satisfaire son besoin d'agitation politique. Il a d'ailleurs rejoint le syndicalisme anarchiste à un moment, en 1941, où celui-ci est déjà en perte de vitesse, en Suède notamment, après la désillusion des révolutions manquées. Mais s'il choisit néanmoins l'anarchisme, et non pas un marxisme doctrinaire ou d'autres 
théories à la mode, comme base de son engagement, c'est parce qu'il y voit aussi la solution aux problèmes de son adolescence, des problèmes qui sont d'ordre individuel, exclusivement.

III

23 Ce choix aura des répercussions, très rapidement, sur sa vie privée et sur sa réflexion politique. C'est dans les cercles anarchistes de Stockholm qu'il rencontre sa future femme, Anne-Marie Götze, la fille d'un anarchiste allemand, Ferdinand Götze, qui avait participé à la guerre d'Espagne et qui venait de se réfugier en Suède. Il lui propose aussitôt le mariage, non pas que ce fût le coup de foudre immédiat, mais plutôt parce qu'il se sent en communion d'idées avec elle - ils sont branchés sur la même longueur et qu'il veut procurer à cette fille, qui est la partenaire privilégiée de ses discussions, une certaine sécurité en lui offrant la nationalité suédoise. Peut-être aussi parce qu'il avait le besoin lui-même de retrouver une famille, un refuge.

24 Anne-Marie, plutôt féministe, n'est pas très enthousiaste à l'idée de se marier, mais se laisse convaincre par des arguments de circonstance. Ayant déjà sa carte de journaliste à Arbetaren, elle deviendra aussi pour Stig Dagerman sa collaboratrice littéraire.

Dans un entretien publié par le Bulletin des Amis de Stig Dagerman, elle le souligne expressément : «Stig n'était pas quelqu'un qui travaillait régulièrement... Il travaillait dès que l'idée lui venait, sans relâche; tout était déjà organisé dans sa tête, mais auparavant nous avions de longues discussions ensemble... Nous parlions beaucoup de l'idée avant qu'il ne se mette à écrire, j'étais pour ainsi dire sa première critique ${ }^{5}$. Il leur arrivait même souvent de tremper leurs plumes dans la même encre, notamment pour des critiques de livres ou de films qu'ils avaient vus ensemble.

C'est sa femme, Anne-Marie, qui a fait prendre conscience à Dagerman de l'immense hypocrisie des forces dites de gauche, en Suède comme en Europe en général, face au révélateur que fut la guerre d'Espagne, avec les tentatives en Catalogne d'autogestion anarcho-syndicaliste dans l'esprit de Bakounine. La Suède socialiste avait même promulgué une loi en 1937 interdisant le volontariat pour l'Espagne, sous peine de prison immédiate. Neutralité obligeait!

Le devoir de l'anarcho-syndicalisme, écrit Dagerman, était de montrer dans quel insoluble dilemme se trouvait alors l'être humain, d'éclairer sa solitude dans une société dite collective, sa détresse morale et les limites de son devoir d'obéissance ${ }^{6}$.

Il s'agit donc bien pour lui, comme nous l'avons déjà souligné, de rechercher des solutions à des problèmes qui sont strictement d'ordre individuel et personnel. Et cela définit en même temps les raisons d'un engagement public qui, en l'occurrence, ne pouvait se concrétiser que par l'écriture. Dagerman entrait en littérature comme on entre en religion, pour satisfaire son besoin d'agir et son besoin d'absolu, son « besoin de consolation », comme il le dira lui-même, avec ses mots à lui.

Il deviendra donc écrivain et journaliste, mais non pas un quelconque pamphlétaire de gauche, un de plus. Son ambition est bien plus subtile et son problème plus complexe. Il a l'étoffe d'un grand écrivain, il le sent, il le sait. Et s'il en doutait encore, la critique, unanime, se charge de l'en persuader dès son premier roman. Il a l'étoffe d'un grand écrivain, et c'est pourquoi il y aura conflit, conflit entre sa conscience sociale qui le porte à l'engagement politique, et sa conscience artistique. Et ce conflit, permanent, 
s'étendant sur la bonne dizaine d'années de sa carrière d'écrivain, fut sans doute une des causes profondes du drame de sa fin. La collaboration de Dagerman au journal Arbetaren, l'organe du mouvement anarcho-syndicaliste en Suède, ne faisait qu'entretenir et aviver cette déchirure intime, comme nous allons le voir.

Ce journal, installé dans Klara Folkets Hus et dont le rédacteur responsable - on ne disait surtout pas «rédacteur en chef », puisque chez les anarchistes il n'y a pas de chef - avait imposé une égalité absolue à tous les journalistes, avait commencé à connaître un certain succès en tant que journal culturel. Surtout à partir de la fin de l'année 1941 où Carl Vennberg avait commencé sa collaboration comme critique littéraire. Vennberg était déjà un très grand poète, mais qui ne partageait guère les vues politiques et l'idéologie du journal. C'était un homme de gauche, mais qui ne s'est jamais déclaré en sympathie avec l'anarchisme.

Entre 1942 et 1944 il avait introduit au journal quelques jeunes auteurs débutants, dont Stig Carlson, Folke Fridell et Stig Dagerman, qui provenaient, du moins pour les deux derniers, du mouvement syndical. Ces jeunes auteurs essayaient de se rapprocher des 40-talister groupés autour de Vennberg, avec Erik Lindegren et Lars Ahlin, pour former un nouveau front de l'esprit et tenter de lancer une nouvelle revue littéraire, Votum.

Ce fut un échec rapide, car tous les 40-talister, ces écrivains des années 1940, étaient loin d'avoir envie tous de s'engager sous la même bannière. En outre, l'éditeur Bonnier allait bientôt publier le numéro un de 40-tal, une revue dont Werner Aspenström était rédacteur en chef, à la grande déception des jeunes anarchistes de Arbetaren, qui se sentaient ainsi, en quelque sorte, doublés.

Cependant, ils se réconcilient assez vite. Stig Dagerman deviendra même secrétaire de 40-tal. Mais leur véritable forum restera le journal syndical, où ils trouvent la possibilité de satisfaire leur besoin de liberté et leur conscience. Lorsqu'on relit à présent cette presse, leur engagement a quelque chose d'émouvant, qui force le respect même. Car malgré leur volonté de coller à la réalité, de peser sur le présent et de transformer le monde, ou peut-être à cause d'elle, on sent qu'ils sont déjà en porte-à-faux, à côté de la plaque en quelque sorte, lorsqu'il s'agit de l'essentiel dans l'immédiat, c'est-à-dire de ce dont un journaliste doit se soucier avant tout. Car l'anarchisme en tant que mouvement politique avait déjà fait faillite.

Werner Aspenström lui avait d'ailleurs réglé son compte d'un trait de plume en écrivant dans 40-tal: "L'anarchisme c'est, tout comme l'esthétisme, une atmosphère agréable à vivre, mais non pas un point de vue à défendre ».

Il est vrai que l'anarchisme, qui demeurait cependant un espoir, un rêve, était devenu alors en Suède un mode de vie autant qu'un engagement politique. Cela convenait d'ailleurs parfaitement à Stig Dagerman, à la fois anarchiste et esthète, mais cela contribuait aussi à faire surgir l'aiguillon de la mauvaise conscience, l'inévitable mauvaise conscience des écrivains Scandinaves, car l'anarchisme continuait aussi pour Stig Dagerman, en son for intérieur, volens nolens, à être un point de vue à défendre, et non seulement un mode de vie.

La conviction anarchiste de Dagerman, cet enfant blessé, ou " enfant brûlé " selon le titre d'un de ses romans, qui trânait derrière lui sa jeunesse et son passé personnel comme une blessure constante, l'amenait à chercher la guérison de sa propre blessure dans la guérisson du monde. Frère lointain de Henri Heine, cet autre " enfant perdu » dans un combat trop lourd pour lui. 

des écrivains, et leur présence à côté d'auteurs que toute la presse de gauche, et notamment $\mathrm{Ny}$ dag, caractérisait comme réactionnaires, provoqua une polémique où de jeunes marxistes convaincus montèrent en première ligne.

43 Dans le conflit qui s'en suivit, et qui fut aussi un conflit de générations, Stig Dagerman prit résolument parti contre les marxistes, et donc contre ceux de sa génération, contrairement à la plupart de ses anciens amis de Arbetaren. Le journal lui-même constate d'ailleurs avec satisfaction, dans plusieurs éditoriaux, que Dagerman ne s'était pas rangé parmi les «kulturbolsjeviker».

Séjournant à l'époque en France, pour préparer un reportage à la manière de celui qu'il venait de publier sur l'Allemagne, Stig Dagerman est profondément troublé, voyant que les deux parties dans ce conflit essaient de le gagner à leur cause. Dans une lettre du 11 
avril 1948 il écrit à son beau-père Ferdinand Götze: "Cette histoire de la Författarförening me fait perdre le sommeil et témoigne d'un chaos intellectuel et spirituel auquel je songe des nuits entières $»^{7}$. Il ne voit de pureté d'intention ni chez les uns ni chez les autres, et déclare même, en conclusion, que le mieux, c'est d'émigrer en Nouvelle-Zélande!

Notons d'ailleurs que ce désir d'émigrer, c'est-à-dire d'échapper, est assez symptomatique pour Dagerman. L'année suivante il partira effectivement à bord d'un bateau de réfugiés à destination de l'Australie. Le prétexte officiel était de trouver parmi les passagers le cadre et l'intrigue d'un scénario de film. Mais déjà au moment de son mariage avec Anne-Marie Götze il avait proposé à celle-ci d'émigrer en Amérique du Sud.

En attendant, et quelques jours seulement après la lettre à son beau-père, il rédige le 17 avril une Lettre ouverte aux écrivains suédois ${ }^{8}$, une lettre de quatre pages datée de Fontainebleau. Dans cette lettre, qu'il faut considérer comme un document très important pour comprendre Dagerman, celui-ci dit qu'il ne veut pas en rajouter à l'hystérie et aux intrigues qui secouent la Författarförening, mais plutôt en tirer les conclusions.

Tout d'abord, écrit-il, il faut réformer cette association sur une base purement syndicale et apolitique, en vue de la défense des seuls intérêts économiques communs des auteurs. Ensuite, et surtout, il faut étudier «la manière de retrouver une santé mentale qui n'est pas du tout favorisée par l'exigence moderne de prendre absolument parti " ${ }^{9}$. Dagerman parle de la véritable catastrophe que représente l'exigence politique de simplification - «det katastrofala förenklingskravet»- c'est-à-dire la tendance manichéenne de séparer les bons des mauvais et de les montrer du doigt. Il s'agit, ajoute-t-il, de s'en tenir tant que l'on peut «à cette forme aiguë de la solitude qui s'appelle la poésie $»^{10}$, une des rares défenses encore possibles contre les psychoses.

Il est extrêmement intéressant de constater ce diagnostic de Dagerman, qui le concerne bien plus lui-même qu'il ne concerne ses confrères. Vouloir concilier littérature et politique, l'art et l'action, ne peut mener qu'à des compromissions, à des capitulations et à des soumissions, à des simplifications catastrophiques. Et au danger de se laisser abuser par les belles paroles et les intentions affichées de tolérance.

Certes, continue-t-il dans cette même lettre, les politiciens sont tout à fait favorables aux écrivains tant qu'ils peuvent en tirer parti... ils peuvent même aller jusqu'à leur garantir une liberté éternelle d'expression. La liberté de parole est évidemment une grande chose, mais la liberté de l'art représente davantage encore, et autant que je puisse m'en rendre compte, c'est surtout cette liberté-là qui est immédiatement menacée ${ }^{11}$.

49 En écrivant cela, Dagerman se défend de vouloir faire la morale à qui que ce soit, il dit même qu'il ne se fait guère d'illusions non plus sur la liberté de l'art, une expression souvent vide de sens. Mais en la plaçant comme idéal au-dessus des autres libertés, il fait en quelque sorte, dans cette lettre, ses adieux à la politique.

Assez curieusement cette lettre - écrite à Fontainebleau, elle fait songer à d'autres " Adieux de Fontainebleau », qui furent aussi de faux adieux à la politique - n'a jamais été publiée. Une copie se trouve en possession d'Anne-Marie Dagerman. Cela montre très clairement le désarroi, les hésitations de son auteur, ne sachant finalement plus quel parti prendre. 
51 La seule chose certaine pour Dagerman, c'est que le climat politique ne devait pas empoisonner le climat intellectuel. Or, il le faisait. Mais au-delà de cette certitude, une certitude immédiate, commençaient toutes les interrogations.

Dagerman essaya de trouver d'autres chemins artistiques, où l'écrivain serait d'abord seul face à sa conscience. Le groupe des écrivains syndicalistes qui avaient collaboré à Arbetaren s'était disloqué. Karl Vennberg s'est même demandé alors si le seul trait commun à tous les 40 -talister n'était pas «en viss politisk hemlöshet» - une certaine absence d'attaches politiques.

53 Tout en continuant sa collaboration à Arbetaren, par fidélité, l'écrivain Stig Dagerman avait déjà commencé à s'engager dans une sorte de passage à vide. Il ne publiera pratiquement plus après 1949, malgré quelques projets de romans dont il n'arriva guère à écrire plus que les quelques pages du début. Les discussions sur l'engagement politique au sein de la Författarförening avaient servi de révélateur. Elles ont certainement eu une influence sur la sorte de paralysie, de sécheresse qui empêchera Dagerman d'écrire et qui avait déjà commencé à faire son œuvre lors de son séjour en France.

Anne-Marie Dagerman dit à ce propos dans son interview donnée au Bulletin des Amis de Stig Dagerman :

Quand nous sommes arrivés en France, j'ai eu de nouveau besoin de ma liberté... C'est à ce moment-là qu'il a commencé à ne plus pouvoir écrire, quand nous nous sommes séparés... Avant d'écrire, il avait besoin d'en parler, d'être réconforté dans son écriture... Quand il n'a plus eu cela, il n'a plus pu écrire ${ }^{12}$.

Sans vouloir, et d'ailleurs sans pouvoir vraiment discuter le rôle que s'attribue ainsi l'ex-épouse de Stig Dagerman, il est certain cependant qu'à partir de cette époque, à partir de leur divorce, celui-ci se trouve presque exclusivement renvoyé, confronté à sa seule conscience d'écrivain.

Non pas la conscience morale des bons apôtres du paradis social qui distinguent aisément entre le bien et le mal - d'ailleurs, sait-il encore vraiment ce qu'est le bien, alors que l'impression de savoir ce qu'est le mal, et de le vivre, est permanente. La conscience à laquelle il est renvoyé est celle qu'il a de sa mission, ou plus simplement de son métier d'écrivain.

Dagerman s'était déjà expliqué là-dessus dans un texte datant de 1945 et intitulé justement L'Écrivain et sa conscience. Il y analyse, dans son style habituel, brillant, imagé et percutant, la nécessité pour l'écrivain de se remettre sans cesse en question, de ne pas se laisser enfermer dans l'outrecuidance de celui qui pense avoir réussi «à enfermer l'infini dans un coffrage en béton armé ». Si un écrivain s'est installé dans le confort intellectuel des certitudes, c'est déjà trop tard pour lui, car « il prend alors ses quartiers au Jardin des plantes, lui qui était jadis un frêle bouleau exposé à tous les vents de la steppe $»^{13}$.

58 Au lieu de ce confort-là, l'écrivain a besoin du choc permanent, pense Dagerman, du choc de la menace qui pèse sur la littérature et qui exige de celle-ci une justification. Une justification?

60 Certains la trouvent facilement, écrit Dagerman, en se réfugiant dans les coteries et les chapelles. Mieux vaut, ajoute-t-il, s'installer dans la forêt des paradoxes.

Comment est-il possible, continue-t-il, de se comporter d'un côté comme si rien au monde n'avait plus d'importance que la littérature, alors que, de l'autre, il est 
impossible de ne pas voir que les gens sont bien obligés de considérer que le plus important pour eux, c'est ce qu'ils gagnent à la fin du mois... L'écrivain ne saurait donc se soustraire au devoir de prise de position. Mais alors se présente un autre problème, qui paraît encore bien plus délicat : comment prendre position ? ... Et voilà sa conscience sociale qui entre en conflit avec sa conscience artistique, et ce conflit-là est insoluble ${ }^{14}$.

Ce texte, daté de 1945 où il s'agissait pour Dagerman, dans l'immédiat, de défendre la liberté de l'art contre les prétentions extravagantes d'un nouveau réalisme, dit marxiste, contient cependant en germe toute la problématique qui éclatera dans la lettre de Fontainebleau et à laquelle Dagerman ne trouvera finalement d'autre solution que la fuite, la mort.

Voulant assumer, par honnêteté envers lui-même - faudrait-il dire aussi par naïveté ? ce conflit entre sa conscience sociale et sa conscience artistique, il lui deviendra de plus en plus difficile de trouver sa place dans une Suède où Ivar Lo-Johansson venait de déclarer après l'abolition du statut des statare ${ }^{15}$ que sa tâche était terminée, alors que pour Dagerman elle n'aurait dû, au contraire, que commencer, la tâche de permettre à des gens qui venaient soudain d'être libérés de comprendre la signification de cette libération ainsi que ses dimensions véritables.

Frei wovon, und frei wozu, selon le mot de Nietzsche. « Ne pas connaître la misère, écrit Dagerman, ne signifie pas ne pas connaître la peur, la peur de devoir faire face à l'absurdité de sa propre existence $»^{16}$.

Comment l'écrivain peut-il aider chacun à assumer sa condition humaine, alors qu'il doit le faire en se gardant de cette littérature des références obligatoires, à laquelle Dagerman voyait se soumettre allègrement et avec la meilleure des consciences tant de ses compagnons de gauche? Comment parler encore à un peuple empesé par des décennies de social-démocratie? Voilà les interrogations qui tourmentent de plus en plus notre auteur. Car il sait que tout le monde attend beaucoup de lui, à commencer par son éditeur, auquel il laissera des dettes considérables à sa mort. Il est devenu célèbre, et les critiques sont à l'affût.

Les critiques lui faisaient mal, toutes, écrit Anne-Marie Dagerman. C'est pourquoi cela fut si dur au cours des dernières années pendant lesquelles il ne pouvait plus écrire ${ }^{17}$.

Oh, il saurait bien encore quoi leur dire, à ses compatriotes. Ses dagsedlar prouvent que la liste de ses indignations n'est pas close. Mais comment le leur dire ? Un sentiment d'impuissance s'empare de lui, progressivement, allant jusqu'à la paralysie intellectuelle, car il ne peut emboucher les trompettes de Jéricho de la cohorte littéraire engagée au service du marxisme orthodoxe.

66 Ce sentiment est encore exacerbé par la vie qu'il est amené à connaître après sa liaison, puis son mariage avec l'actrice Anita Björk, dans la belle villa de banlieue qu'il partage avec elle. Une vie facile, de l'autre côté de la rue, où il pouvait donner libre cours à sa passion des belles voitures, où la tentation du luxe était permanente. Une vie qui lui plaisait, assurément, et qui lui permettait de cultiver ses allures à la James Dean, auquel le liait son engouement pour le cinéma et une ressemblance qui allait jusqu'à une étonnante ressemblance physique. Leur mort même empruntera les mêmes chemins. Dagerman s'est suicidé dans sa voiture, en 1954, et un an plus tard James Dean, dont l'idéal était de "vivre vite, mourir jeune et faire un beau cadavre », s'est tué dans un accident de voiture. 
Dagerman est mort sans laisser de dernier message, sans s'expliquer sur les raisons de son geste. A moins qu'on ne considère qu'il l'avait déjà fait quatre ans auparavant dans sa lettre à Ragnar Svanström citée en introduction.

Ayant déjà fait antérieurement plusieurs tentatives de suicide, peut-être en avait-il parlé avec Anita Björk. Mais celle-ci, alors au sommet de sa célébrité, évoluait dans un autre monde, trop différent de celui que Stig avait connu dans sa jeunesse et pour lequel il s'était promis d'œuvrer, de s'engager. Le goût de la pauvreté, qui avait été le goût de son enfance, s'était effacé de ses lèvres. Il est probable qu'un psychanalyste aurait décelé chez lui une sorte de schizophrénie évolutive, un fossé se creusant lentement entre ce qu'il avait été, et dont il se montrait jaloux, et ce qu'il ne pouvait plus être. Il avait d'ailleurs consenti, sur les conseils de quelques amis, à suivre une cure dans une maison de santé, mais s'était montré assez rétif, finissant rapidement par refuser tout traitement.

Pour concilier les exigences contraires de son engagement social en tant qu'écrivain, dans lesquelles résidait précisément le caractère ambigu de cet engagement, Dagerman s'est mis après 1949 à la recherche d'activités nouvelles, de nouveaux moyens d'expression comme la radio ou le théâtre. Sans succès également. Car ces derniers se heurtaient aussi, tout comme l'écriture romanesque, aux deux exigences de plus en plus inconciliables de la conscience sociale et de la conscience artistique. Il y avait chez Dagerman un besoin d'honnêteté fondamental sur lequel il ne transigeait pas - ce qu'il appelle sans doute son innocence - de sorte qu'il ne cherchait à esquiver aucune des deux exigences. Il est peu probable donc que ce soit uniquement le sentiment de s'être renié en passant du côté des riches, comme on le dit parfois, qui ait provoqué chez lui la sécheresse et le désir de mort.

Tenant à analyser l'angoisse, sa propre angoisse, il s'est livré à de dangereuses expériences, tel un enfant qui veut absolument s'approcher jusqu'au bord du ravin. D'où ses tentatives de suicide apparemment bien dosées, comme chez ces drogués qui connaissent la juste dose qu'il leur faut. Jusqu'au jour où tout bascule, parce qu'il n'a plus la force de regarder ce vide ni d'assumer la responsabilité de ses propres questions, de ses "rêves immuables", l'anarcho-syndicalisme, la justice, la pensée libertaire, ces valeurs dans la défense desquelles il avait mis un radicalisme entier et qui devaient se heurter immanquablement à la question de leur fondement.

On ne peut se battre indéfiniment pour des abstractions, comme le remarque très justement Régis Boyer dans un article consacré à Stig Dagerman et le vide ontologique. Analysant cet infléchissement irréversible qui conduit Dagerman d'un amour intense de la vie au pied du mur, ou au bord du vide, R. Boyer écrit : « Les buts que Dagerman s'était assignés auraient exigé des assises métaphysiques ou religieuses bien plus solides que celles qu'il s'était données $»^{18}$.

2 Mais c'est là un autre aspect, différent et sans doute plus fondamental encore, de la problématique de notre auteur.

Dans un papier qu'on a retrouvé dans un de ses tiroirs après sa mort, Dagerman écrit :

Tout homme connaît un moment affreux où la vie semble se lasser de lui et, avec une grimace, le laisser seul dans un vide qui ne recèle même plus de désir de mort... 
Si cet homme est écrivain, les mots se décomposent en lettres et l'alphabet devient un cimetière aux vingt-six pierres tombales sous lesquelles la littérature est enterrée ${ }^{19}$.

Un autre écrivain, bien oublié aujourd'hui, qui était pourtant Prix Nobel de littérature et qui vivait en Suède à la même époque que Dagerman, il s'agit de Nelly Sachs, avait employé à peu près les mêmes images métaphoriques, en parlant dans un de ses poèmes de l'alphabet perdu - «das verlorene Alphabet». Mais Nelly Sachs, qui ne souffrait pas d'un vide, mais plutôt d'un trop-plein ontologique, a complété le titre de son poème, le poème lui-même et finalement le sens de sa propre vie d'écrivain, en annonçant que l'alphabet perdu pouvait être et avait été retrouvé - «Das verlorene und wieder gerettete Alphabet». Chez Dagerman il n'y a pas eu d'alphabet retrouvé, rien que les vingt-six pierres tombales sous lesquelles la littérature est enterrée, sans rémission.

Nous savons que Dagerman lui-même n'a pas été dupe de son mal. Des analyses différentes, qui divergent dans leur méthode et leur inspiration, convergent toutes sur ce point. Et plutôt que de vouloir le guérir au prix des compromissions, il a préféré garder son innocence.

Ci-gît un écrivain suédois

tombé pour rien.

Son crime était l'innocence,

oubliez-le souvent.

\section{NOTES}

1. - R. Svanström, « Souvenirs sur Stig Dagerman », in Plein Chant, 31-32, 1986, p. 78.

2. - Ibid.

3. - S. Dagerman, «L'Anarchisme et moi », in Plein Chant, 31-32, 1986, p. 60.

4. - Cf. Stig Claesson, Bönder, Vem aiskar Yngve Frej, entre autres.

5. - Anne-Marie Dagerman, in Bulletin des Amis de Stig Dagerman, 1, 1990, p. 12.

6. - Stig Dagerman, L'Anarchisme et moi, cf. supra.

7. - Stig Dagerman, correspondance, Archives A.M. Dagerman.

8. - S. Dagerman, Öppet brev tili de flesta svenska författare, 17.4.1948, Archives A.M. Dagerman.

9. - Ibid.

10. - Ibid.

11. - Ibid.

12. - A.M. Dagerman, In Bulletin des Amis de Stig Dagerman, 1, 1990, p. 14.

13. - Stig Dagerman, «L'Écrivain et la conscience », in 40-tal, 1940, 6. Cf. aussi Plein Chant 31-32, 1986, p. 91.

14. - Ibid., p. 95.

15. - Les statare étaient des ouvriers agricoles dont le statut, proche du servage, fut officiellement aboli en 1945.

16. - Stig Dagerman, «Le rôle de la littérature est de faire comprendre le sens de la liberté », in Plein Chant, cf. supra, p. 101.

17. - A.M. Dagerman, op. cit., p. 15. 
18. - Régis Boyer, «Stig Dagerman et le vide ontologique», in Le Texte et l'idée, 3/1988, p. 151.

19. - Cité par O. Lagercrantz, in Stig Dagerman, Norstedt 1958, p. 181.

\section{RÉSUMÉS}

"Je quitte des rêves immuables et des liaisons inutiles... une position irrésolue face aux problèmes de notre temps ». Ces mots désabusés, Stig Dagerman les adresse à son éditeur quatre années exactement avant son suicide réussi. Et pourtant, la célébrité lui avait souri, il est sollicité de toutes parts, mais il a le sentiment d'être écartelé entre des exigences contradictoires. Pour comprendre le drame qui se noue et qui aboutit à une insupportable stérilité, il faut partir de ce qui a été à la base de sa vocation d'écrivain, son engagement anarchiste.

Le jeune Dagerman est un militant convaincu, son idéologie libertaire sous-tend ses premières œuvres. Mais il y aura progressivement naissance d'un conflit entre sa conscience sociale qui le pousse à l'action et sa conscience artistique. Ne pouvant échapper à la nécessité d'un choix, il parle dans une lettre ouverte aux écrivains suédois de la véritable catastrophe que représente l'exigence politique manichéenne... La seule défense est de «s'en tenir à cette forme aiguë de la solitude qui s'appelle la poésie». Mais ne voulant renier ses engagements, ne pouvant les concilier avec sa conscience d'artiste, il aboutit dans l'impasse. Certes, la liste de ses indignations n'est pas close ; mais comment les dire ? Dagerman est mort sans laisser de dernier message.

«Ich verlasse unwandelbare Traume und unnütze Bindungen... eine unschlüssige Stellung den Problemen unserer Zeit gegenüber...». Genau vier Jahre vor Seinem Selbstmord schrieb Dagerman diese blasierten Worte an seinen Verleger. Und dies zu einer Zeit, wo er doch berühmt war. Er hatte aber das Gefühl, zwischen entgegengesetzten Ansprüchen hin und her gerissen zu sein, und sein persönliches Drama tritt immer deutlicher zu Tage. Eine mögliche Erklärung kann nur von dem anarchistischen Engagement ausgehen, das seinem Schriftstellerberuf zu grunde lag.

Schon als Gymnasiast beginnt er politisch tätig zu sein, in der anarchistischen Front in Stockholm. Seine ersten Werke sind ganz von dieser Lebensund Weltauffassung durchdrungen. Aber bald entsteht ein Konflikt zwischen seinem sozialen und seinem ästhetischen Gewissen, er sieht sich vor eine unumgängliche Wahl gestellt. In einem offenen Brief an die schwedischen Schriftsteller schreibt er, es sei eine wirkliche Katastrophe, immer wieder auf die manicheistische Forderung der Politik zu stossen. Nur ein einziger Ausweg bleibe noch, «jene hohe Form der Einsamkeit, die man Poesie nennt». Da er aber weiter an seinem anarchistischen Engagement festhält, spitzt sich der Widerspruch mit seinem ästhetischen Gewissen zu, und seine Feder droht, zu erlahmen. Gewiss, es fehlt seiner Entrüstung nicht an dem nötigen Material, aber welches Kleid dafür? Stig Dagerman ist weggegangen ohne letzte Botschaft.

\section{AUTEUR}

\section{GEORGES UEBERSCHLAG}

Université Charles-de-Gaulle - Lille III 